\title{
Unification of intermediate energy hadron spectra with small energy losses
}

\author{
R.J. Peterson ${ }^{\mathrm{a}}$ \\ University of Colorado, Boulder, CO 80309-0390, USA
}

\begin{abstract}
Proton beams near $1 \mathrm{GeV}$ may serve as prolific generators of secondary neutrons, with some neutron energies near the beam energy. These very energetic secondary neutrons in turn may generate further reactions in thick samples. Direct measurement of the cross sections for $\mathrm{GeV}$-scale neutrons is difficult, leading to uncertainties in flux calculations for many proposed applications. The present work uses a simple kinematic reaction model to form a scaling response suited to compare $(\mathrm{p}, \mathrm{nx})$ spectra for a wide range of beam energies, angles and target nuclei, to enable comparisons, interpolations and perhaps extrapolations of these difficult data. If the approximations of the method below are valid, one could also relate $(\mathrm{p}, \mathrm{nx})$ and easier $(\mathrm{p}, \mathrm{px})$ measurements.
\end{abstract}

The goal of this work is to compare a wide range of (p,px) and $(\mathrm{p}, \mathrm{nx})$ spectra with small energy losses for intermediate energy proton beams by way of scaling relations found to be successful for the simple (e,ex) reaction. This method must assume incoherent, quasifree factorization of the nuclear response and simple scattering of the proton from a single bound nucleon. Free (off-shell) singly differential cross sections are used for the reaction, and the response is taken to be proportional to the number of nucleons seen once and only once by the projectile, as calculated in the eikonal Glauber manner. This computation uses in-medium total cross sections for protonnucleon scattering.

For low energy losses, the most useful scaling is that due to Bjorken, with the kinematic variable $x_{B}=\left(q^{2}-\omega^{2}\right) / 2 M \omega$, with q the lab frame 3-momentum transfer from the projectile, $\omega$ the lab frame energy loss of the projectile and $M$ the free nucleon mass [1]. Free proton-neutron charge exchange would occur at $x_{B}=1$, and the variable $x_{B}$ can be understood as the fraction of the total nuclear momentum found in that one single struck nucleon. Important corrections for charge and binding energy are used, as presented for the (p,px) spectra in [2]. The Bjorken response is then

$$
F_{B}=\frac{d^{2} \sigma / d \omega d \Omega}{A_{e f f} d \sigma / d \Omega} \quad \frac{2 M \omega^{2}}{\omega^{2}+q^{2}},
$$

using measured doubly-differential cross sections. All published (p,nx) spectra to be found, from 345 to $1600 \mathrm{MeV}$, have been transformed in this fashion into responses $F_{B}$. If these are the same for combinations of beam energy, scattering angle and nuclear target, several forms of scaling will have been identified. Once shown to be valid, one can use these relations to interpolate, extrapolate or evaluate the cases of interest. Since the determination of the cross sections for high energy neutrons is most difficult, these are the cases presented here, at large values of $x_{B}$. Production of such high energy neutrons can lead to secondary or cascade reactions in thick samples, making these scaling relations an important tool for many applications of accelerator-produced neutrons.

\footnotetext{
${ }^{a}$ Presenting author,

e-mail: jerry.peterson@colorado.edu
}

The test case is for $800 \mathrm{MeV}$ protons on lead, with one reliable (p,px) work [3] and six (p,nx) examples [4-9]. Data at similar angles are compared in figure 1, from $x_{B}=1$ (for free p-p scattering) to $x_{B}=5.5$, corresponding to an energy loss $\omega=50 \mathrm{MeV}$ at $30^{\circ}$. The curves show the (p,px) responses from lead at $800 \mathrm{MeV}$ over the same range of angles [2,3]. The $30^{\circ} \mathrm{Pb}(\mathrm{p}, \mathrm{nx})$ responses do not all agree with one another near $x_{B}=1$, but scatter around the $\mathrm{Pb}(\mathrm{p}, \mathrm{px})$ dot-dashed result. The $20^{\circ} \mathrm{Pb}(\mathrm{p}, \mathrm{nx})$ responses (dashed) match those for (p,px) at $x_{B}=1$, but fall below those at larger $x_{B}$. Overall, the trends for the two classes of reaction change in the same way with increasing angle, but more strongly for the charge exchange case.

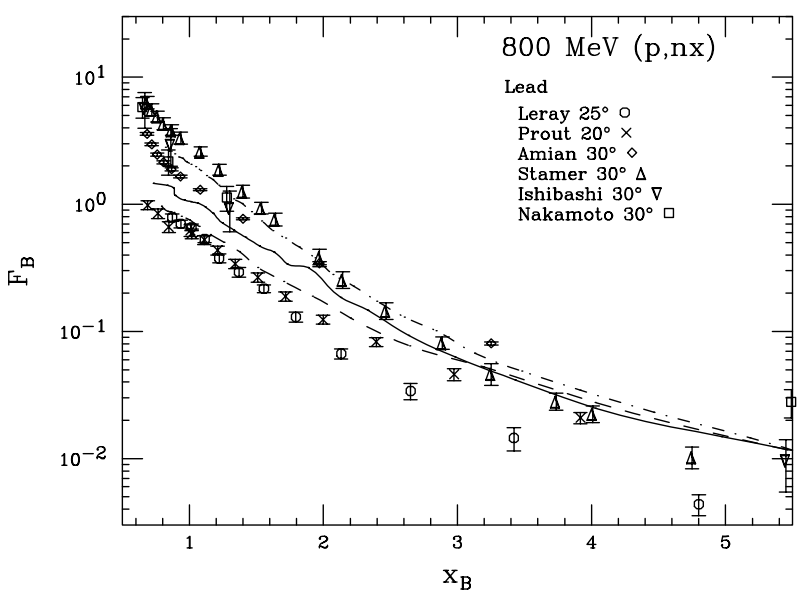

Fig. 1. The world supply of $800 \mathrm{MeV} \mathrm{Pb}(\mathrm{p}, \mathrm{nx})$ spectra at 20-30 degrees is shown in the Bjorken format, using $N_{\text {eff }}=7.20$ out of 126 as the number of neutrons seen once and only once for the charge exchange reaction [4-9]. The curves show the corresponding $\mathrm{Pb}(\mathrm{p}, \mathrm{px})$ responses, increasing as the scattering angle changes from $20,25,30^{\circ}$.

Most proposed targets for accelerator-produced neutrons use heavy nuclei, so figure 2 collects Bjorken responses for such cases at four beam energies at similar angles. Overall, these agree, demonstrating the validity of the Glauber method used to evaluate $N_{e f f}$. 


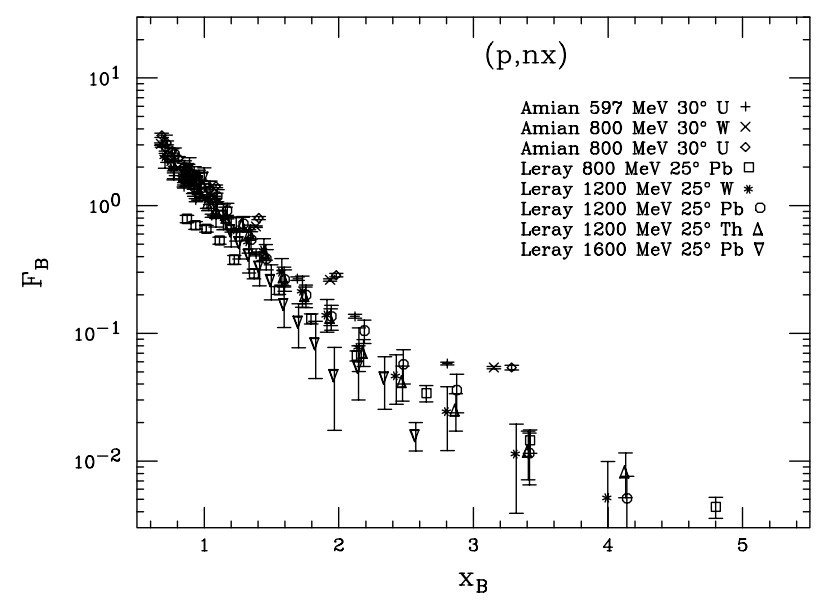

Fig. 2. The important cases of (p,nx) spectra from heavy nuclei at a range of proton beam energies are collected $[4,6,11]$ for energy losses less than those for free single nucleon scattering (at $x_{B}=1$ ). For example, at $1200 \mathrm{MeV}$ and $25^{\circ}$ the neutron energies at $x_{B}=2,3$, 4 and 5 are 1032, 1086, 1114 and $1131 \mathrm{MeV}$, respectively.

The most severe assumption made in this analysis is the factorization, such that the responses should be independent of beam energy or scattering angle. Figure 3 shows the responses for the case of carbon, used by the largest number of experiments, as a function of $q[4-6,10,11]$. Scaling would be obtained if these responses were the same. Figure 3 uses $F_{B}$ interpolated for $x_{B}=2$ and 3 . Also shown in figure 3 are the Bjorken responses computed in the same way for transverse electron scattering [12], also evoking an isovector nuclear response. Note one example of aluminum [6], not carbon, as the target. Except for this case, the charge exchange responses are seen to scale. The solid curves show the best fits to the similar carbon (p,px) responses [2]. The charge exchange responses at energy losses less than those for free scattering are seen to be weaker than for the scattering without charge exchange.

There are several successful cases of scaling among (p,nx) spectra in this Bjorken system at the small angles shown here, enough to enable at least some applications. Comparison to (p,px) responses is hampered by the lack of suitable experimental data at beam energies above $800 \mathrm{MeV}$. If it could be shown that the best cases of scattering and charge exchange reactions obeyed some simple scaling comparisons, one could substitute the simpler scattering data for the very difficult determinations of high eneregy neutron cross sections. The

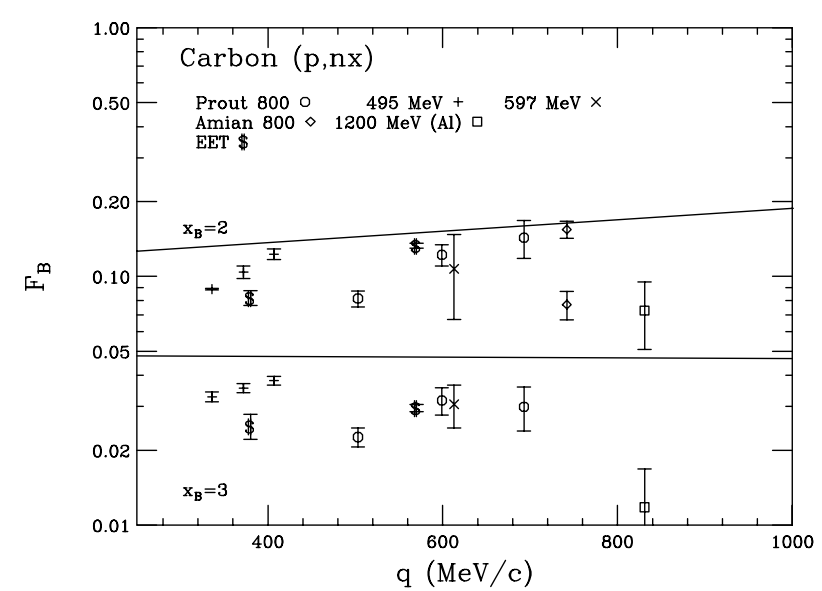

Fig. 3. Bjorken responses $F_{B}$ for $\mathrm{C}(\mathrm{p}, \mathrm{nx})[4-6,11]$ are collected as interpolated for $x_{B}=2$ and 3 for a range of proton beam energies and angles, to test the basic scaling hypothesis. Also shown is the (isovector) tranverse electron scattering response [12]. Except for the one case with aluminum, not carbon [6], these responses do scale, although at a level weaker than that shown by the solid lines, taken as the best fit to the $\mathrm{C}(\mathrm{p}, \mathrm{px})$ responses [2].

hadron-induced nuclear responses can also provide important results on nucleon momentum distributions and correlations within nuclei, enhancing the results obtained from electron scattering, as in [1].

This work was supported in part by the US DOE.

\section{References}

1. K.S. Egiyan et al., Phys. Rev. C 68, 014313 (2003); Phys. Rev. Lett. 96, 082501 (2006).

2. R.J. Peterson (to appear in Nucl. Phys. A, 2007).

3. R.E. Chrien et al., Phys. Rev. C 21, 1014 (1980).

4. W.B. Amian et al., Nucl. Sci. Eng. 112, 78 (1992).

5. D.L. Prout et al., Phys. Rev. C 52, 228 (1995).

6. S. Leray et al., Phys. Rev. C 65, 044621 (2001).

7. S. Stamer et al., Phys. Rev. C 47, 1647 (1993).

8. K. Ishibashi et al., Nucl. Sci. Technol. 34, 529 (1997).

9. T. Nakamoto et al., Nucl. Sci. Technol. 32, 827 (1995).

10. X.Y. Chen et al., Phys. Rev. C 47, 2159 (1993).

11. W.B. Amian et al., Nucl. Sci. Eng. 115, 1 (1993).

12. P. Barreau et al., Nucl. Phys. A 402, 515 (1983). 